\title{
Effects of sporadic transthyretin amyloidosis frequently on the gallbladder and the correlation between amyloid deposition in the gallbladder and heart
}

Shojiro Ichimata ( $\square$ ichimata@med.u-toyama.ac.jp )

University of Toyama

Yukiko Hata

University of Toyama

Naoki Nishida

University of Toyama

\section{Research Article}

Keywords: amyloidosis, gallbladder, gastrointestinal tract, heart, transthyretin

Posted Date: April 6th, 2021

DOI: https://doi.org/10.21203/rs.3.rs-397058/v1

License: (c) (i) This work is licensed under a Creative Commons Attribution 4.0 International License. Read Full License 


\section{Abstract}

The aim of the study is to evaluate the clinicopathological features of cholecystic ATTR deposition in patients with cardiac involvement, investigate the correlation of amyloid deposition severity in the gallbladder and the heart, and compare its prevalence in the gallbladder and other organs. Fifty patients with sporadic ATTR amyloidosis were identified. Of these, we evaluated 15 patients who underwent gallbladder sampling accurately. Among 10 patients (67\%) with cholecystic deposition, six exhibited detectable deposition in the hematoxylin and eosin-stained specimens, and all of them displayed obstructive vascular deposition (VD). The severity of gall bladder VD was statistically correlated with that of cardiac VD and atrial interstitial deposition (ID). Additionally, all patients exhibiting cholecystic ID displayed severe ventricular and atrial IDs. In visceral organs excluding the heart, amyloid deposition was commonly observed in the lungs (93\%), followed by the gastrointestinal tract (47\%$80 \%)$, liver $(60 \%)$, and periosteal tissues (53\%). The involvement of gallbladder was prevalent and comparable to that of the gastrointestinal tract. Moreover, the severity of cholecystic deposition was correlated with that of cardiac deposition. Therefore, pathologists should be aware that sporadic ATTR amyloidosis is a common condition and should not be overlooked.

\section{Introduction}

Transthyretin (ATTR) amyloidosis is one of the major causes of cardiac amyloidosis (CA), which is the leading cause of death in patients with systemic amyloidosis ${ }^{1,2}$. ATTR amyloidosis can be hereditary or wild-type (ATTRwt). The frequency of the latter form, previously called senile systemic amyloidosis increases with aging ${ }^{1-3}$. Recently, the efficacy of tafamidis for ATTR CA was confirmed, making the disease a treatable condition ${ }^{4}$. Because this drug is expected to have greater benefit when administered early in the disease course, diagnosis of ATTR amyloidosis at an early stage has become an important issue.

Gallstones are one of the most common abdominal conditions requiring hospitalization in developed countries ${ }^{5}$. Since the frequency of gallstones increases with age, the number of cholecystectomy is expected to increase with the increase in the elderly population, owing to greater life expectancies in recent years ${ }^{5}$. Moreover, because of advances in surgical techniques and perioperative care, the use of cholecystectomy is increasing in older adults ${ }^{6}$. Therefore, because the frequency of sporadic ATTR amyloidosis, including ATTRwt and very late-onset hereditary ATTR, increases with age and it can affect systemic organs ${ }^{7,8}$, the frequency of the incidental discovery of ATTR deposition in cholecystectomy specimens should be increasing. However, cholecystic involvement in systemic amyloidosis, including ATTR amyloidosis, is considered extremely rare, and only a few cases have been reported $^{9-15}$. As these reports contain only one or a small number of cases, the frequency and clinicopathological features of sporadic cholecystic ATTR deposition remain unclear. Moreover, since there is an association between the frequency of ATTR deposition in the adipose tissue and the severity of ATTR $\mathrm{CA}^{8}$, there may be an association in the severity between the heart and other organs including the gallbladder.

We previously performed histochemistry and immunohistochemistry to detect clinically undiagnosed diseases including ATTR $\mathrm{CA}$ and neurodegenerative diseases without case selection in serial forensic autopsy-based cases, including several healthy or asymptomatic participants $8,16,17$. Such an analysis is useful for examining the clinicopathological features and incidence of clinically undiagnosed diseases. In addition, these patients were considered potentially suitable for cholecystectomy because no individual was in a bedridden state and all had reasonable physical functioning in daily life at the time of death. Furthermore, for the same reason, such patients may have been good candidates for tafamidis therapy. To the best of our knowledge, no studies have evaluated cholecystic ATTR deposition in such patients.

In this study, 15 forensic autopsy cases aged 70 years or older with clinically undiagnosed ATTR CA were used to evaluate the frequency and clinicopathological features of sporadic cholecystic ATTR deposition. In addition, we compared the prevalence of amyloid deposition between the gallbladder and several organs subjected to biopsy to diagnose amyloidosis, including the lungs, liver, kidneys, gastrointestinal (GI) tract, bone marrow (BM), and periosteal tissues ${ }^{18}$.

\section{Results}


In total, 50 patients of sporadic ATTR CA were identified (2.7\% of all patients, $6.2 \%$ of these were of age $\geq 70$ years). No patient had a familial history of polyneuropathy or systemic amyloidosis. In addition, no patient had been clinically diagnosed with CA. Among the 50 patients, the gallbladder was correctly sampled in 16 patients. Of these, one patient who died from burns and who exhibited significantly decreased congophilia was excluded from the evaluation. The patients' clinical features are summarized in Table 1. All patients were at least 70 years old, and the mean patient age was $87.5 \pm 6.2$ years (range, 78-97; median, 87). The male-to-female ratio was 6:9. In 6 of 15 patients, the cause of death was considered CA (40\%). Of these six patients, four (67\%) had signs of CA including edema or chronic heart failure. The detailed pathological findings of the heart in Cases 1-10 were reported previously. ${ }^{8}$

Table 1

Clinical summary of the patients and the histopathological findings in the gallbladder and heart

\begin{tabular}{|c|c|c|c|c|c|c|c|c|c|}
\hline \multirow[t]{2}{*}{ No } & \multirow[t]{2}{*}{ Age } & \multirow[t]{2}{*}{ Sex } & \multirow{2}{*}{$\begin{array}{l}\text { Manner/Cause } \\
\text { of death }\end{array}$} & \multirow[t]{2}{*}{ Complications } & \multicolumn{2}{|l|}{ Gallbladder } & \multicolumn{3}{|l|}{ Heart } \\
\hline & & & & & HE (V/I) & $\begin{array}{l}\text { CR } \\
(\mathrm{V} / \mathrm{I})\end{array}$ & Ventricle & Atrium & Vessel \\
\hline 1 & 80 & M & Nat/tumor & $\mathrm{N} / \mathrm{A}$ & indefinite/indefinite & $0 / 0$ & $2+$ & $2+$ & 0 \\
\hline 2 & 80 & M & Acci/traffic & $\mathrm{HT}$ & indefinite/indefinite & $1+/ 0$ & $2+$ & $1+$ & $1+$ \\
\hline 3 & 97 & $\mathrm{~F}$ & Nat/CA & HT, Edema & detectable/indefinite & $2+/+$ & $3+$ & $3+$ & $2+$ \\
\hline 4 & 84 & $\mathrm{~F}$ & Acci/Bleeding & CRF & indefinite/indefinite & $1+/ 0$ & $1+$ & $2+$ & $1+$ \\
\hline 5 & 85 & M & Acci/hypothermia & $\mathrm{DM}$ & detectable/indefinite & $2+/ 0$ & 0 & 0 & $2+$ \\
\hline 6 & 87 & $M$ & Nat/CA & $\mathrm{HT}, \mathrm{CHF}$ & indefinite/indefinite & $1+/+$ & $3+$ & $3+$ & $1+$ \\
\hline 7 & 85 & $\mathrm{~F}$ & Sui/Drowning & $\mathrm{HL}, \mathrm{HT}$ & indefinite/indefinite & $0 / 0$ & $1+$ & $1+$ & 0 \\
\hline 8 & 94 & $\mathrm{~F}$ & Nat/CA & $\mathrm{AP}, \mathrm{HT}, \mathrm{CHF}$ & detectable/indefinite & $2+/+$ & $3+$ & $3+$ & $2+$ \\
\hline 9 & 96 & $\mathrm{~F}$ & Acci/fall & $\mathrm{HL}, \mathrm{HT}$ & detectable/indefinite & $2+/+$ & $1+$ & $3+$ & $2+$ \\
\hline 10 & 81 & $\mathrm{~F}$ & Nat/CA & $\mathrm{DM}, \mathrm{HL}$ & indefinite/indefinite & $0 / 0$ & $1+$ & $2+$ & 0 \\
\hline 11 & 90 & $\mathrm{~F}$ & Acci/hypothermia & None & detectable/indefinite & $2+/ 0$ & $2+$ & $2+$ & $1+$ \\
\hline 12 & 89 & $M$ & Acci/drowning & None & indefinite/indefinite & $1+/ 0$ & $2+$ & $2+$ & $1+$ \\
\hline 13 & 78 & M & Nat/CA & $\begin{array}{l}\text { CRF, DM, } \\
\text { edema }\end{array}$ & indefinite/indefinite & $0 / 0$ & $2+$ & $2+$ & 0 \\
\hline 14 & 90 & $\mathrm{~F}$ & Acci/drowning & $\mathrm{HT}$ & indefinite/indefinite & $0 / 0$ & $1+$ & $1+$ & 0 \\
\hline 15 & 97 & $\mathrm{~F}$ & Nat/CA & None & detectable/indefinite & $2+/+$ & $3+$ & $3+$ & $1+$ \\
\hline
\end{tabular}

\section{Histopathological Findings In The Gallbladder And Heart}

Among 15 patients with ATTR CA, 10 patients (67\%) displayed cholecystic ATTR deposition. Of these patients, amyloid deposits were detectable in HE-stained specimens in six patients (detectable group). The deposits were mainly observed on the vessels, and all patients exhibiting obstructive VD had detectable amyloid deposition in HE-stained specimens (Fig. 3A; $P<0.01$ ). In addition, obstructive VD in the heart was identified only in the detectable group (Fig. 3B; $P=0.011$ ). However, although the incidence of severe ventricular ID was higher in the detectable group than in the indefinite group ( $50 \%$ vs. $11 \%$, respectively), these differences did not reach statistical significance (Fig. 3C; $P=0.23$ ). By contrast, the incidence of severe atrial ID was 
significantly higher in the detectable group (Fig. 3D, $P=0.023$ ). No significant differences in the pathological findings, such as mucosal necrosis and severity of inflammatory change, were identified between the cases with and without obstructive VD.

Cholecystic ID was identified in five patients (cholecystic ID-positive group), whereas the remaining 10 patients comprised the cholecystic ID-negative group. Four of five with cholecystic ID were in the detectable group. However, because ID foci were small and faint, none could be identified in HE-stained specimens. Severe cardiac ID was identified only in the cholecystic ID-positive group in both the ventricle and atrium (Fig. 3E, F, P < 0.01). However, although the incidence of death from CA was higher in the cholecystic ID-positive group than in the cholecystic ID-negative group ( $80 \%$ vs. $20 \%)$, these differences did not reach statistical significance $(P=0.09)$.

\section{Histopathological Findings In Visceral Organs Other Than The Heart}

The results of the histopathological observation are presented in Table 2. Amyloid deposition was most frequently observed in the lungs (93\%), followed by the intestine (80\%), liver (60\%), colon (54\%), esophagus (53\%), periosteal tissues (53\%), and stomach (47\%). The mean prevalence in the GI tract was $57 \%$ (30/53 specimens). In the periosteal tissues, amyloid deposition was most frequently observed in connective tissue other than ligaments (47\%). Conversely, renal involvement was identified only in one patient (7\%). Amyloid deposition was limited to vessels in the liver and kidneys, and VD was predominant in the GI tract. Meanwhile, ID was predominant in the lungs and periosteal tissues. No amyloid deposition was identified in the BM stroma. 
Table 2

Prevalence of ATTR amyloid deposition in other visceral organs other than the heart

\begin{tabular}{|c|c|c|c|c|c|c|c|c|c|}
\hline \multirow[t]{2}{*}{ Case } & \multirow{2}{*}{$\begin{array}{l}\text { Lung } \\
(\mathrm{V} / \mathrm{I})\end{array}$} & \multirow{2}{*}{$\begin{array}{l}\text { Liver } \\
(\mathrm{V} / \mathrm{I})\end{array}$} & \multirow{2}{*}{$\begin{array}{l}\text { Kidney } \\
(\mathrm{V} / \mathrm{I})\end{array}$} & \multicolumn{4}{|c|}{ Gastrointestinal tracts (V/I) } & \multicolumn{2}{|l|}{ Bone } \\
\hline & & & & Esophagus & Stomach & Intestine & Colon & $\begin{array}{l}\text { BM stroma } \\
(\mathrm{N} / \mathrm{I})\end{array}$ & РОТ (V/L/C) \\
\hline 1 & $1+/+$ & $0 / 0$ & $0 / 0$ & $0 / 0$ & $0 /+$ & $\mathrm{N} / \mathrm{A}$ & $\mathrm{N} / \mathrm{A}$ & $0 / 0$ & $0 / 0 /+$ \\
\hline 2 & $0 /+$ & $1+/ 0$ & $0 / 0$ & $0 / 0$ & $0 / 0$ & $1+/ 0$ & $0 / 0$ & $0 / 0$ & $0 / 0 / 0$ \\
\hline 3 & $1+/+$ & $2+/ 0$ & $1+/ 0$ & $2+/+$ & $1+/+$ & $2+/+$ & $2+/+$ & $0 / 0$ & $1+/+/+$ \\
\hline 4 & $1+/+$ & $0 / 0$ & $0 / 0$ & $1+/ 0$ & $1+/ 0$ & $1+/+$ & $1+/ 0$ & $0 / 0$ & $0 / 0 / 0$ \\
\hline 5 & $0 /+$ & $1+/ 0$ & $0 / 0$ & $1+/ 0$ & $0 / 0$ & $1+/ 0$ & $2+/ 0$ & $0 / 0$ & $2+/ 0 /+$ \\
\hline 6 & $1+/+$ & $1+/ 0$ & $0 / 0$ & $1+/ 0$ & $0 / 0$ & $1+/ 0$ & $\mathrm{~N} / \mathrm{A}$ & $0 / 0$ & $1+/+/+$ \\
\hline 7 & $0 /+$ & $0 / 0$ & $0 / 0$ & $0 / 0$ & $0 / 0$ & $0 / 0$ & $0 / 0$ & $0 / 0$ & $0 / 0 / 0$ \\
\hline 8 & $1+/+$ & $2+/ 0$ & $0 / 0$ & $2+/+$ & $2+/+$ & $1+/ 0$ & $1+/ 0$ & $0 / 0$ & $1+/ 0 /+$ \\
\hline 9 & $1+/ 0$ & $1+/ 0$ & $0 / 0$ & $1+/ 0$ & $1+/ 0$ & $2+/ 0$ & $1+/ 0$ & $0 / 0$ & $0 /+/ 0$ \\
\hline 10 & $0 /+$ & $0 / 0$ & $0 / 0$ & $0 / 0$ & $0 / 0$ & $0 / 0$ & $0 / 0$ & $0 / 0$ & $0 / 0 / 0$ \\
\hline 11 & $1+/+$ & $1+/ 0$ & $0 / 0$ & $1+/ 0$ & $1+/ 0$ & $2+/+$ & $2+/ 0$ & $0 / 0$ & $0 / 0 / 0$ \\
\hline 12 & $1+/+$ & $1+/ 0$ & $0 / 0$ & $0 / 0$ & $0 / 0$ & $\mathrm{~N} / \mathrm{A}$ & $0 / 0$ & $0 / 0$ & $0 / 0 / 0$ \\
\hline 13 & $0 /+$ & $0 / 0$ & $0 / 0$ & $0 / 0$ & $0 / 0$ & $\mathrm{~N} / \mathrm{A}$ & $0 / 0$ & $0 / 0$ & $0 /+/+$ \\
\hline 14 & $0 / 0$ & $0 / 0$ & $0 / 0$ & $0 / 0$ & $0 / 0$ & $\mathrm{~N} / \mathrm{A}$ & $0 / 0$ & $0 / 0$ & $0 / 0 / 0$ \\
\hline 15 & $1+/+$ & $1+/ 0$ & $0 / 0$ & $2+/ 0$ & $1+/ 0$ & $\mathrm{~N} / \mathrm{A}$ & $2+/ 0$ & $0 / 0$ & $1+/+/+$ \\
\hline $\begin{array}{l}\text { PR (All } \\
[\%])\end{array}$ & $\begin{array}{l}14 / 15 \\
(93)\end{array}$ & $\begin{array}{l}9 / 15 \\
(60)\end{array}$ & $\begin{array}{l}1 / 15 \\
(7)\end{array}$ & $\begin{array}{l}8 / 15(53) \\
(53 / 13)\end{array}$ & $\begin{array}{l}7 / 15 \\
(47)\end{array}$ & $\begin{array}{l}8 / 10 \\
(80)\end{array}$ & $\begin{array}{l}7 / 13 \\
(54)\end{array}$ & $\begin{array}{l}0 / 15(0) \\
(0 / 0)\end{array}$ & $\begin{array}{l}8 / 15(53) \\
(33 / 33 / 47)^{\star}\end{array}$ \\
\hline$(\mathrm{V} / \mathrm{l}$ & $(60 / 87)$ & $(60 / 0)$ & $(7 / 0)$ & & $(40 / 20)$ & $(80 / 30)$ & $(54 / 8)$ & & \\
\hline \multicolumn{10}{|c|}{$\begin{array}{l}\text { Abbreviations: BM, bone marrow; N/A, not available; POT, periosteal tissues; PR, positive rate; } \mathrm{V} / \mathrm{I} \text {, vessel/interstitium; } \mathrm{V} / \mathrm{L} / \mathrm{C} \text {, } \\
\text { vessel/ligament/connective tissue other than ligaments }\end{array}$} \\
\hline
\end{tabular}

\section{Discussion}

In this study, we clearly found that two-thirds of patients with clinically undiagnosed sporadic ATTR CA exhibited cholecystic involvement. This result is consistent with a previous autopsy-based study ( $3 / 4$ cases, $75 \%)^{15}$. The prevalence of sporadic ATTR deposition among patients $\geq 80$ years old has been reported as approximately $9-12 \%$ in Japan ${ }^{8,15,19}$ and $20-25 \%$ in Western 
countries $^{20-22}$. Therefore, it is estimated that sporadic ATTR deposition is present in approximately $6-8 \%$ and $13-17 \%$ of cholecystectomy materials obtained from patients older than 80 years in Japan and Western countries, respectively. Thus, it should be noted that cholecystic ATTR deposition may not be rare in elderly patients, and most instances of deposition might have been overlooked.

This study clearly demonstrated that the severity of ATTR deposition in the gallbladder is correlated with that in the heart. Therefore, we considered that the severity of ATTR deposition in the heart could be predicted from the amount of deposition in the organs other than the heart. In particular, the presence of cholecystic ID was indicative of severe cardiac involvement. Of note, most patients with cholecystic ID exhibited obstructive VD that was easily identified in HE-stained specimens. In addition, in the gallbladder, ATTR deposits were mainly observed on the vessels. Thus, to ensure that latent cholecystic ATTR deposition is not overlooked, careful observation of the vessels appears important ${ }^{9}$.

Conversely, we could not identify most instances of non-obstructive VD using HE-stained specimens. This may be related to the fact that gallbladder specimens are prone to degeneration because of autolysis and inflammation, which make it difficult to observe the tissue architecture. In addition, histopathological diagnoses in the gallbladder are generally made using only HE staining unless cancer or another specific disease is clinically suspected. Thus, latent ATTR deposition could be easily missed. This may one reason why cholecystic involvement in systemic amyloidosis is considered rare ${ }^{9-14}$. Although the severity of cardiac involvement in patients with only non-obstructive VD is considered mild to moderate, the presence of sporadic ATTR deposition in the heart, regardless of its amount, might be a risk factor for sudden cardiac death ${ }^{8}$. Thus, we recommend CR staining to evaluate the presence or absence of amyloid deposition in cholecystectomy specimens obtained from patients aged 80 years or older, especially those with signs of sporadic ATTR amyloidosis including edema, heart failure, arrhythmia (especially atrial fibrillation), and carpal tunnel syndrome (CTS) 1,2,23,24. Moreover, we should assess whether cholecystic ATTR deposition is associated with the increased postoperative mortality after cholecystectomy.

According to the aforementioned correlation in the severity of deposition between the gallbladder and heart, patients with only non-obstructive VD are considered to have early-stage CA. Recently, the efficacy of tafamidis against cardiac ATTR amyloidosis was confirmed, making the disease treatable ${ }^{4}$. This drug is expected to have greater benefit when administered early in the disease course. Therefore, these patients are considered good candidates for treatment with tafamidis because their physical condition and cardiac function were considered sufficient to permit cholecystectomy ${ }^{4}$. However, tafamidis is one of the most expensive cardiovascular drugs to reach the market, and it may be impractical to provide this treatment to all patients with cardiac ATTR amyloidosis ${ }^{25,26}$. Therefore, we should discuss the future management of ATTR amyloidosis that is incidentally detected in surgical specimens including samples from cholecystectomy.

The prevalence of amyloid deposition in several organs in this study was consistent with previous findings ${ }^{15,27,28}$. The prevalence of cholecystic involvement was comparable with that in the GI tract, perhaps because both have similar tissue architecture. Contrarily, renal involvement was rare, and no glomerular and interstitial involvement was identified. Therefore, although Sekijima et al. reported that renal dysfunction is a common clinical finding in patients with ATTRwt amyloidosis in a nationwide survey in Japan ${ }^{29}$, direct deposition of ATTR fibrils in the kidneys might not be the major cause of symptoms. Meanwhile, no amyloid deposition was identified in the BM stroma. Taken together with previous findings ${ }^{28}$, the presence of amyloid deposition in the BM stroma may be a diagnostic clue for immunoglobulin light chain amyloidosis.

Ligament involvement is extremely common in ATTRwt amyloidosis $23,24,30,31$. In particular, CTS has been reported as the most common initial symptom, and patients may present with symptoms of CTS 5-15 years prior to cardiac impairment ${ }^{23}$. However, only one-third of patients with ATTR CA exhibited ligament involvement in this study. There are two possibilities for this discrepancy. First, some ligaments are more prone to ATTR amyloid deposition, similarly as the susceptibility to deposition varies by organ ${ }^{18,32}$. The second possibility is that ATTR deposition is affected by the presence or absence of degeneration and inflammation. Because previous reports evaluated surgical materials obtained from symptomatic sites, degeneration and inflammation should be present ${ }^{24,30,31}$. Conversely, we evaluated the ligaments with no observed inflammation and degeneration, and thus, amyloid deposition might have been missed. 
In addition to a certain level of bias in our study population, this study was also limited by the lack of clinical information for some patients including the presence or absence of CTS or spinal stenosis, mainly because of the lack of severe clinical symptoms. Moreover, we could not perform TTR gene testing and prevent the inclusion of patients with elderly-onset hereditary ATTR amyloidosis ${ }^{6}$. Therefore, we described the cases as sporadic ATTR amyloidosis. Furthermore, because cholecystic specimens obtained at autopsy are degenerated in almost all cases and these specimens are often of no macroscopic pathological significance, they are not regularly sampled in our department, and we could evaluate them in 15 of 50 patients.

In summary, we demonstrated that sporadic ATTR amyloidosis frequently involves the gallbladder, and the severity of cholecystic amyloid deposition is correlated with that of the heart. Because ATTR deposition in the gallbladder is often indefinite in $\mathrm{HE}$-stained specimens, we recommend CR staining for cholecystectomy specimen obtained from elderly patients, especially those aged 80 years or older with signs of CA. Because ATTR amyloidosis has become a treatable disease, clinicians and pathologists must recognize the importance of accurate diagnosis, especially in the early stage. Pathologists should be aware that sporadic ATTR amyloidosis is not a rare disease and evidence of its involvement that may be present within routine pathological specimens.

\section{Methods}

\section{Subjects}

We reviewed the archives of all 2213 medicolegal autopsies in our department between 2008 and June 2020. Among these, we evaluated the specimens from 1826 patients for whom all organs, including the brain, could be sampled. The clinical histories of the patients were obtained from their families and the records of police examinations. When there was a history of a hospital visit, the medical records were provided by the primary physician.

This study was approved by the Ethical Committee of Toyama University (12020006) and was performed per the ethical standards established in the 1964 Declaration of Helsinki and updated in 2008.

\section{Pathological Examination}

The methods of pathologic examination of hearts in our department were described in a previous report ${ }^{33}$. All specimens were fixed in $20 \%$ buffered formalin and routinely embedded in paraffin. Then, $4-\mu \mathrm{m}$-thick sections were cut, stained with hematoxylin and eosin (HE), and subjected to immunohistochemistry. In addition, 6- $\mu$ m-thick sections were cut and used for Congo red (CR) staining. The screening and confirmation methods of CA were described previously ${ }^{8}$. After the histopathological confirmation, immunohistochemical typing was performed using antibodies for transthyretin (EPR3219, 1:2000; Abcam plc, Cambridge, UK) ${ }^{8}$, anti-kappa (H16-E, 1:500; DB Biotech, Kosice, Slovak Republic) ${ }^{34}$, and lambda light chains (polyclonal, 1:1000; Abcam plc). Furthermore, if a medical history of hemodialysis or chronic inflammatory diseases was present, immunohistochemistry using 32-microglobulin antibodies (polyclonal, 1:3000; GeneTex) or amyloid A (mc1, 1:5000; Dako, Glostrup, Denmark), respectively, was additionally performed. Retrieval of all antigens, excluding anti-amyloid A, was performed using 100\% formic acid for 1 min. Immunostaining was performed using the Leica Bond-IV automation and Leica Refine detection kits (Leica Biosystems, Bannockburn, IL, USA), according to the manufacturer's instructions. Subsequently, all sections were counterstained with hematoxylin.

\section{Histopathological Evaluation}

We evaluated the extent of vascular (VD) and interstitial amyloid deposition (ID) semi-quantitatively by light microscopy using specimens subjected to $\mathrm{CR}$ staining ${ }^{8,35}$. In the gallbladder, before evaluating CR-stained specimens, we evaluated whether the deposition was recognizable on HE staining (detectable or indefinite). In addition, the results of ATTR deposition in the gallbladder and heart were also confirmed using immunostaining for transthyretin. Additionally, if venous amyloid deposition displayed weak congophilia was identified in the GI tract, we performed immunohistochemistry for transthyretin to identify epidermal growth factor-containing fibulin-like extracellular matrix protein 1-derived amyloid deposition ${ }^{36,37}$. The representative findings in the gallbladder and heart are presented in Figs. 1 and 2, respectively. The severity of VD was graded as follows: 0 , 
none; $1+$, non-obstructive ( $<75 \%$ reduction in the luminal cross-sectional area); and $2+$, obstructive ( $\geq 75 \%$ reduction). Meanwhile, ID, excluding the heart, was graded as follows: 0, none; and + , present. Additionally, ID in the heart was semiquantitatively graded as follows: 0 , none; $1+$, mild, focal ( $<5 \%$ of the myocardial area); $2+$ moderate, multifocal $(\geq 5 \%$ but $<$ $50 \%$ ); and $3+$, severe, extensive ( $\geq 50 \%$ ). Ventricular amyloid deposition was measured on both sides of ventricles and the ventricular septum, and atrial amyloid deposition was measured at the atrial septum. Bone and the surrounding tissues were sampled from the ribs, and periosteal tissues were subdivided into vessel, ligament, and connective tissues other than the ligament, including muscle and fatty tissue. All histopathological grades were determined at the site at which the most amyloid deposits were observed at $\times 100$ magnification.

\section{Statistical analysis}

The relationships of the extent of cholecystic VD with those of ID and deposition in the heart were assessed using Fisher's exact test. Differences between groups were considered significant at $P<0.05$.

\section{Abbreviations}

ATTR, amyloid transthyretin; BM, bone marrow; CA, cardiac amyloidosis, CR, Congo red; CTS, carpal tunnel syndrome; GI, gastrointestinal; ID, interstitial deposition; VD, vascular deposition

\section{Declarations}

\section{Acknowledgments:}

The authors are grateful to Mr. Noboru Onozuka, Ms. Syuko Matsumori, Ms. Miyuki Maekawa, and Mr. Osamu Yamamoto for their technical assistance.

\section{Author contributions:}

SI designed the study, took photographs, and drafted the manuscript. YH collected the patient's medical history and reviewed the manuscript. NN collected the patient's medical history and critically reviewed the manuscript.

\section{Competing Interests:}

No potential competing interests was reported by the authors.

\section{Funding disclosure:}

This work was supported in part by JSPS KAKENHI Grant Numbers JP20K18979 to S. I.

\section{References}

1. Wechalekar, A. D., Gillmore, J. D. \& Hawkins, P. N. Systemic amyloidosis. The Lancet 387, 2641-2654 (2016).

2. Nakagawa, M., Sekijima, Y., Tojo, K. \& Ikeda, S. High prevalence of ATTR amyloidosis in endomyocardial biopsy-proven cardiac amyloidosis patients. Amyloid 20, 138-140 (2013).

3. Benson, M. D. et al. Amyloid nomenclature 2020: update and recommendations by the International Society of Amyloidosis (ISA) nomenclature committee. Amyloid 27, 217-222 (2020).

4. Maurer, M. S. et al. Tafamidis treatment for patients with transthyretin amyloid cardiomyopathy. Engl. J. Med. 379, 10071016 (2018).

5. Bray, F. et al. Increased incidence of cholecystectomy related to gallbladder disease in France: Analysis of 807,307 cholecystectomy procedures over a period of seven years. Visc. Surg. 156, 209-215 (2019).

6. Loozen, C. S., van Ramshorst, B., van Santvoort, H. C. \& Boerma, D. Early cholecystectomy for acute cholecystitis in the elderly population: a systematic review and meta-analysis. Surg. 34, 371-379 (2017). 
7. Yamashita, T. et al. Genetic and clinical characteristics of hereditary transthyretin amyloidosis in endemic and non-endemic areas: experience from a single-referral center in Japan. Neurol. 265, 134-140 (2018).

8. Ichimata, S., Hata, Y., Hirono, K., Yamaguchi, Y. \& Nishida, N. Clinicopathological features of clinically undiagnosed sporadic transthyretin cardiac amyloidosis: a forensic autopsy-based series. Amyloid, 1-9; 10.1080/13506129.2021.1882979 (2021).

9. Ichimata, S. et al. Spheroid-type transthyretin amyloidosis in the gallbladder: A possible histopathological diagnostic clue to prevent overlooking cholecystic latent amyloid deposition. Pathol. Case Rep. 21, 200407; 10.1016/j.ehpc.2020.200407 (2020).

10. Matsuda, S. et al. An Unusual Case of Acute Cholecystitis with Amyloidosis: A Case Report and Literature Review. Med. 58, 803-807 (2019).

11. Um, Y. J., Kim, H. A., Jung, J. H., Cho, H. \& Kang, J. K. A case of amyloidosis presenting as chronic cholecystitis, misdiagnosed as polymyalgia rheumatica. Korean J. Gastroenterol. 68, 49-53 (2016).

12. Tirotta, D. \& Durante, V. Uncommon localization of amyloidosis in gallbladder: Description of a case and brief literature review. Hepatol. 10, 227-232 (2011).

13. Kwon, A. H., Tsuji, K., Yamada, H., Okazaki, K. \& Sakaida, N. Amyloidosis of the gallbladder mimicking gallbladder cancer. Gastroenterol. 42, 261-264 (2007).

14. Kim, S. H. et al. Abdominal amyloidosis: spectrum of radiological findings. Radiol. 58, 610-620 (2003).

15. Ueda, M. et al. Clinicopathological features of senile systemic amyloidosis: an ante- and post-mortem study. Pathol. 24, 1533-1544 (2011).

16. Yoshida, K. et al. Incipient progressive supranuclear palsy is more common than expected and may comprise clinicopathological subtypes: a forensic autopsy series. Acta Neuropathol. 133, 809-823 (2017).

17. Nishida, N., Yoshida, K., Hata, Y., Arai, Y. \& Kinoshita, K. Pathological features of preclinical or early clinical stages of corticobasal degeneration: a comparison with advanced cases. Appl. Neurobiol. 41, 893-905 (2015).

18. Abe, R. et al. Distribution of amyloidosis subtypes based on tissue biopsy site - Consecutive analysis of 729 patients at a single amyloidosis center in Japan. Int. 71, 70-79 (2021).

19. Shiozaki, T., Sato, N., Hayashi, T., Kobayashi, K. \& Asamura, H. Wild-type ATTR amyloidosis may be associated with unexpected death among the elderly. Med. (Tokyo) 41, 101634 (2019).

20. Falk, R. H. Senile systemic amyloidosis: are regional differences real or do they reflect different diagnostic suspicion and use of techniques? Amyloid 19, 68-70 (2012).

21. Tanskanen, M. et al. Senile systemic amyloidosis affects $25 \%$ of the very aged and associates with genetic variation in alpha2-macroglobulin and tau: a population-based autopsy study. Med. 40, 232-239 (2008).

22. Cornwell, G. G., 3rd, Murdoch, W. L., Kyle, R. A., Westermark, P. \& Pitkanen, P. Frequency and distribution of senile cardiovascular amyloid. A clinicopathologic correlation. J. Med. 75, 618-623 (1983).

23. Fosbol, E. L. et al. Association of carpal tunnel syndrome with amyloidosis, heart failure, and adverse cardiovascular outcomes. Am. Coll. Cardiol. 74, 15-23 (2019).

24. Nakagawa, M. et al. Carpal tunnel syndrome: a common initial symptom of systemic wild-type ATTR (ATTRwt) amyloidosis. Amyloid 23, 58-63 (2016).

25. Kittleson, M. M. et al. Cardiac amyloidosis: evolving diagnosis and management: A scientific statement from the American Heart Association. Circulation 142, e7-e22 (2020).

26. Kazi, D. S. et al. Cost-effectiveness of tafamidis therapy for transthyretin amyloid cardiomyopathy. Circulation 141, 12141224 (2020).

27. Fine, N. M. et al. Yield of noncardiac biopsy for the diagnosis of transthyretin cardiac amyloidosis. J. Cardiol. 113, 17231727 (2014).

28. Javidiparsijani, S. \& Picken, M. M. Should the reporting of bonemarrow positivity for amyloid be revised? A critical assessment based on 66 biopsies from a single institution. Pathol. Lab. Med., 10.5858/arpa.2019-0324-0A (2020). 
29. Sekijima, Y. et al. First nationwide survey on systemic wild-type ATTR amyloidosis in Japan. Amyloid 25, 8-10, doi:10.1080/13506129.2017.1409706 (2018).

30. Yanagisawa, A. et al. Knee osteoarthritis associated with different kinds of amyloid deposits and the impact of aging on type of amyloid. Amyloid 23, 26-32 (2016).

31. Yanagisawa, A. et al. Amyloid deposits derived from transthyretin in the ligamentum flavum as related to lumbar spinal canal stenosis. Pathol. 28, 201-207 (2015).

32. Ichimata, S. et al. Usefulness of gastroduodenal biopsy in the differential diagnosis of systemic AH amyloidosis from systemic AL amyloidosis. Histopathology 73, 230-239 (2018).

33. Hata, Y. et al. Minimal inflammatory foci of unknown etiology may be a tentative sign of early stage inherited cardiomyopathy. Pathol. 32, 1281-1290 (2019).

34. Hoshii, Y., Nanbara, H., Cui, D., Takahashi, M. \& Ikeda, E. Immunohistochemical examination of Akappa amyloidosis with antibody against adjacent portion of the carboxy terminus of immunoglobulin kappa light chain. Mol. Morphol. 45, 124128 (2012).

35. Larsen, B. T. et al. Correlation of histomorphological pattern of cardiac amyloid deposition with amyloid type: a histological and proteomic analysis of 108 cases. Histopathology 68, 648-656 (2016).

36. Tasaki, M. et al. A novel age-related venous amyloidosis derived from EGF-containing fibulin-like extracellular matrix protein 1. Pathol. 247, 444-455 (2019).

37. Dao, L. N. et al. The novel form of amyloidosis derived from EGF-containing fibulin-like extracellular matrix protein 1 (EFEMP1) preferentially affects the lower gastrointestinal tract of elderly females(a). Histopathology, 10.1111/his.14276 (2020).

\section{Figures}




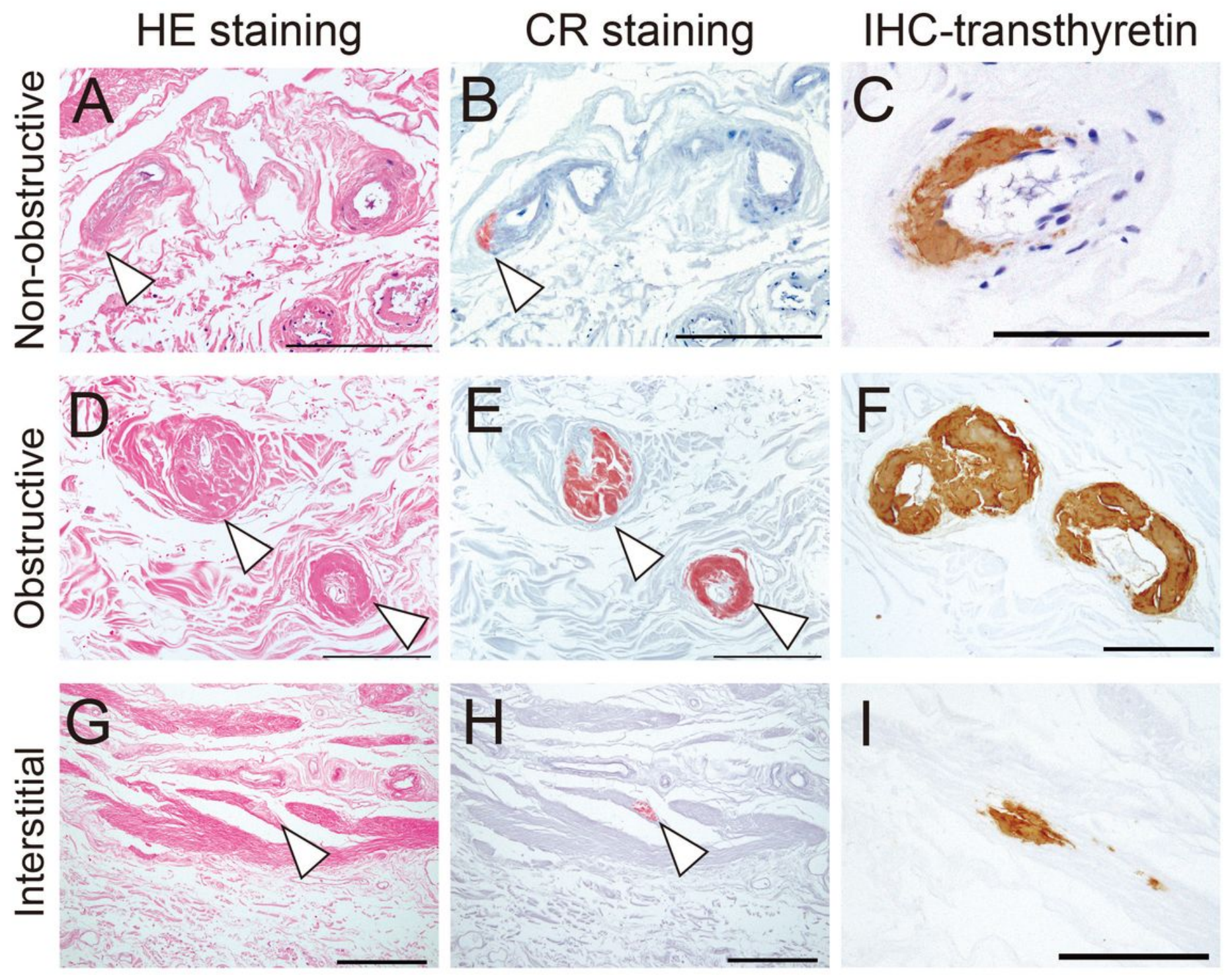

Figure 1

Histopathological findings in the gallbladder. (A-C) Non-obstructive vascular deposition (VD). (D-F) obstructive VD. (G-I) Interstitial deposition. Samples were examined using hematoxylin and eosin (HE) staining ( $A, D, G)$, Congo red (CR) staining (B, E, $H)$, and immunohistochemistry $(I H C)$ for transthyretin $(C, F, I)$. Non-obstructive VD was indefinite in the HE-stained specimen (A, arrowhead), whereas obstructive VD was easily identified in the HE-stained specimen (D, arrowhead). Interstitial deposition was indefinite in the HE-stained specimen (G, arrowhead). Scale bar $=100(A-F, I)$ and $200 \mu \mathrm{m}(G, H)$. 
CR staining
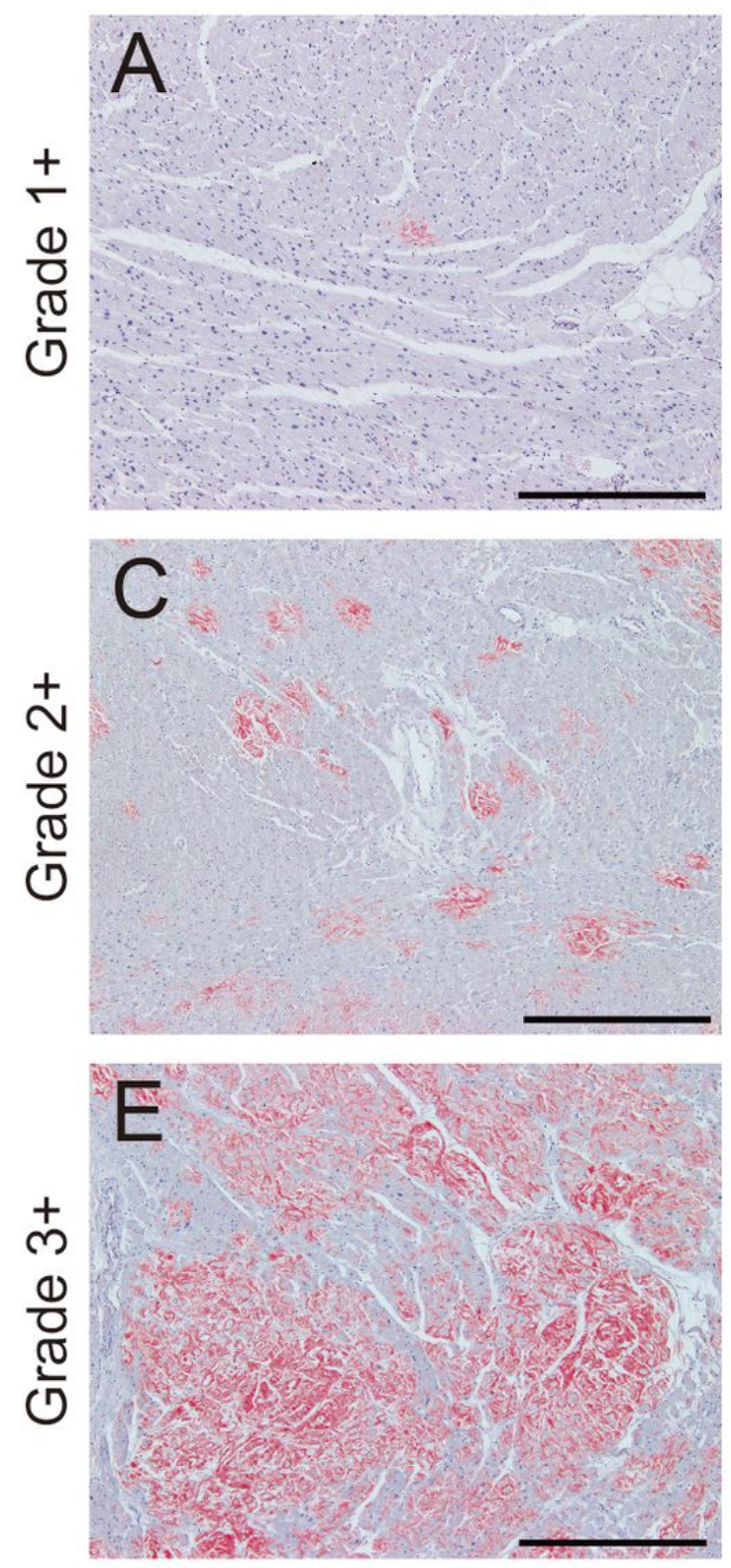

IHC-transthyretin
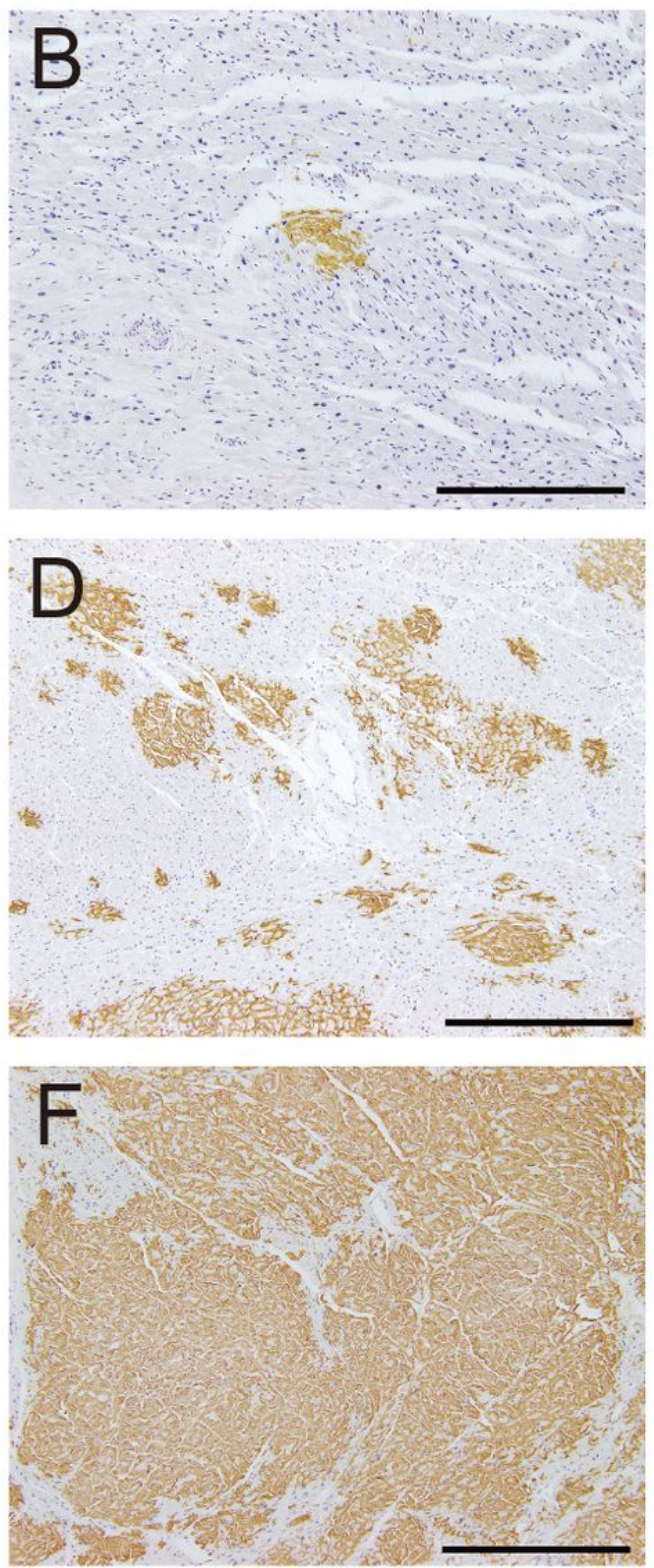

\section{Figure 2}

Histopathological grading in the heart. (A, B) Grade 1+, mild, focal ( $<5 \%$ of the myocardial area). (C, D) Grade $2+$, moderate, multifocal ( $\geq 5 \%$ but $<50 \%$ of the myocardial area). (E, F) Grade $3+$, severe, extensive ( $\geq 50 \%$ of myocardial area). Samples were submitted to Congo red staining ( $A, C, E)$ or immunohistochemistry $(I H C)$ for transthyretin $(B, D, F)$. Scale bar $=500 \mu m(A-F)$. 
A Cholecystic VD grade

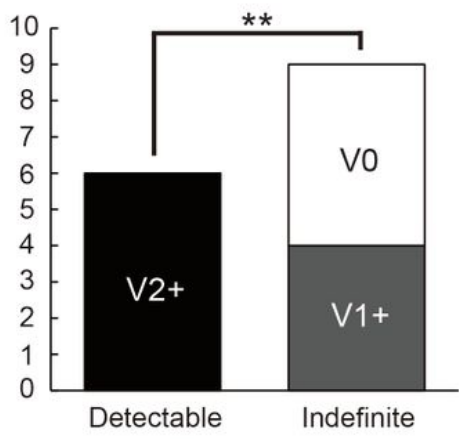

C
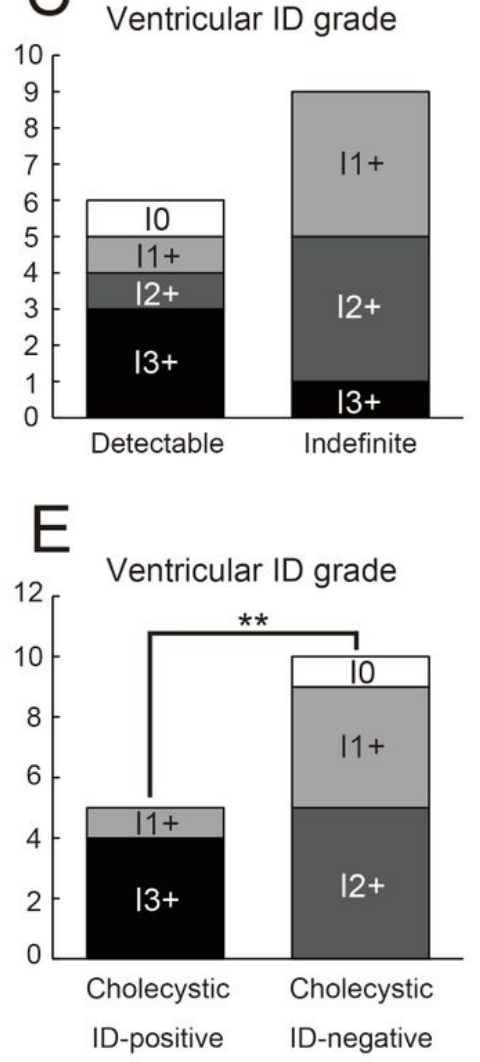

B Cardiac VD grade

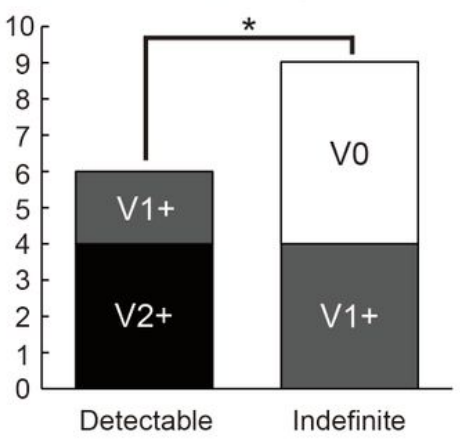

D Atrial ID grade
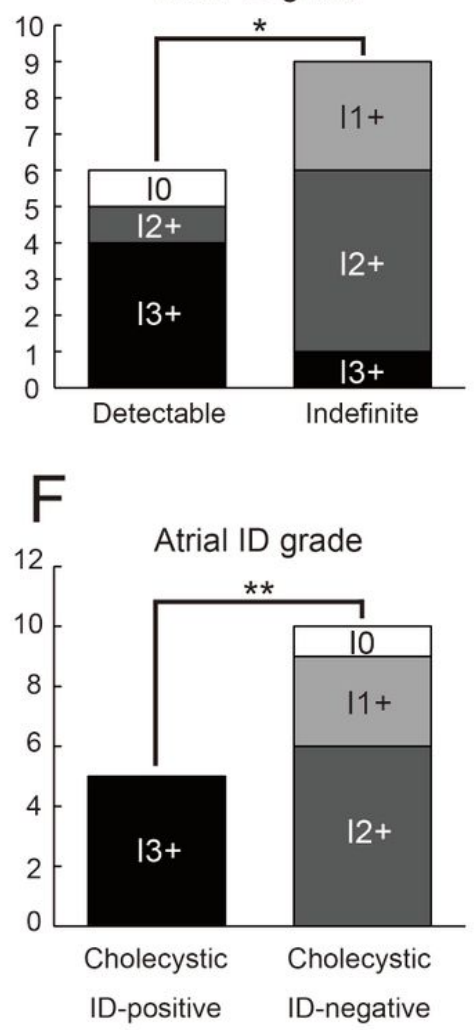

\section{Figure 3}

The severity of amyloid deposition in the gallbladder and heart. (A, B), Cholecystic and cardiac vascular deposition (VD) grade in the detectable and indefinite groups $\left({ }^{\star \star} p<0.01,{ }^{\star} p=0.011\right)$. (C, D), Ventricular and atrial interstitial deposition (ID) grade in the detectable and indefinite groups $\left({ }^{\star} p=0.023\right)$. $(E, F)$, Ventricular and atrial ID grade in the cholecystic ID-positive and cholecystic ID-negative groups $\left({ }^{\star *} p<0.01\right)$. 